\title{
Mechanical stretch increases CCN2/CTGF expression in anterior cruciate ligament-derived cells
}

Yoshiaki Miyake ${ }^{\mathrm{a}, \mathrm{b}}$, Takayuki Furumatsu ${ }^{\mathrm{a}, *}$, Satoshi Kubota ${ }^{\mathrm{b}}$, Kazumi Kawata ${ }^{\mathrm{b}}$,Toshifumi Ozaki ${ }^{\mathrm{a}}$, Masaharu Takigawa ${ }^{\mathrm{b}}$

a) Department of Orthopaedic Surgery, Science of Functional Recovery and Reconstruction, Okayama University Graduate School of Medicine, Dentistry, and Pharmaceutical Sciences

b) Department of Biochemistry and Molecular Dentistry

Okayama University Graduate School of Medicine, Dentistry, and Pharmaceutical Sciences

2-5-1 Shikata-cho, Kita-ku, Okayama, Japan

* Correspondence to: Dr. Takayuki Furumatsu, Dept. of Orthopaedic Surgery, Science of Functional Recovery and Reconstruction, Okayama University Graduate School of Medicine, Dentistry, and Pharmaceutical Sciences, 2-5-1 Shikata-cho, Kita-ku, Okayama 700-8558, Japan.

Tel.: 81-86-235-7273; Fax: 81-86-223-9727; E-mail: matino@md.okayama-u.ac.jp

Running title: Roles of CCN2/CTGF in ACL 


\section{ABSTRACT}

Anterior cruciate ligament (ACL)-to-bone interface serves to minimize the stress concentrations that would arise between two different tissues. Mechanical stretch plays an important role in maintaining cell-specific features by inducing CCN family 2/connective tissue growth factor (CCN2/CTGF). We previously reported that cyclic tensile strain (CTS) stimulates $\alpha 1(\mathrm{I})$ collagen (COL1A1) expression in human ACL-derived cells. However, the biological function and stress-related response of CCN2/CTGF were still unclear in ACL fibroblasts. In the present study, CCN2/CTGF was observed in ACL-to-bone interface, but was not in the midsubstance region by immunohistochemical analyses. CTS treatments induced higher increase of CCN2/CTGF expression and secretion in interface cells compared with midsubstance cells. COL1A1 expression was not influenced by CCN2/CTGF treatment in interface cells despite CCN2/CTGF stimulated COL1A1 expression in midsubstance cells. However, CCN2/CTGF stimulated the proliferation of interface cells. Our results suggest that distinct biological function of stretch-induced CCN2/CTGF might regulate region-specific phenotypes of ACL-derived cells.

Keywords: CCN2/CTGF; anterior cruciate ligament; cyclic tensile strain; collagen; ligament-to-bone interface 


\section{Introduction}

$\mathrm{CCN}$ family $2 /$ connective tissue growth factor $(\mathrm{CCN} 2 / \mathrm{CTGF})$ is a multifunctional regulator of cellular proliferation, differentiation, and tissue regeneration [1]. Ccn2/ctgf-null mice are perinatal lethal, showing severe chondrodisplasia characterized by deficient extracellular matrix (ECM) production, impaired endochondral ossification, and reduced growth plate angiogenesis [2]. These phenotypes indicate that CCN2/CTGF plays an essential role in skeletal development during embryogenesis. In the adult, the expression of CCN2/CTGF is associated with injury repair and inflammation [3]. Several studies have reported that CCN2/CTGF promotes the proliferation of vascular endothelial cells [4], chondrocytes [5], osteoblastic cells [6], and periodontal ligament cells [7]. In addition, CCN2/CTGF is known to stimulate cellular differentiation and ECM synthesis as well in these cells [5-7]. However, the expression pattern and function of CCN2/CTGF in human ligament tissues remain unclear.

Anterior cruciate ligament (ACL) injuries usually occur in the femoral attachment of ACL that contains interface cells [8]. Those cells in ACL-to-bone insertion have more chondrocytic phenotypes compared with midsubstance cells in the middle part of ACL $[9,10]$. ACL-to-bone interface has a specific structure called fibrocartilaginous enthesis that is composed of four different regions; dense fibrous connective tissue (ligament), uncalcified fibrocartilage, calcified fibrocartilage, and subchondral bone $[11,12]$. Fibrocartilaginous enthesis serves to minimize the stress concentrations that would arise between the two different tissues, such as extensible tendon/ligament and rigid bone $[13,14]$. We have previously reported that cyclic tensile strain (CTS) stimulates $\alpha 1(\mathrm{I})$ collagen gene (COL1A1) expression in both interface and midsubstance cells derived from human ACL [15]. In this literature, stretching treatment induced higher increase of COL1A1 expression in interface cells rather than in midsubstance cells [15]. These findings suggest that mechanical stretch has a crucial role in maintaining the homeostasis of ACL and ACL-to-bone interface [16].

The ligaments have stress-oriented structures of collagen bundles [17]. Type I collagen is the main component of ECM in the ligaments [18]. Type II and III collagens play important roles for the maturation of ligament-to-bone interface zones $[12,19]$. Biomechanical studies have shown that the 
length of human ACL is exposed to $12 \%$ elongation at 90 degrees of knee flexion [17]. Human cadaveric ACL-to-bone units fail at a mean strain level of $19 \%$ by a material testing system [20]. In human ACL cells, uni-axial 10\% CTS increases the gene expression of type I and III collagens mediated by the autocrine secretion of transforming growth factor (TGF)- $\beta 1$ [21]. However, the biological function and mechanical stretch-related response of CCN2/CTGF are still unclear in ACL fibroblasts. Effect of mechanical strain on CCN2/CTGF expression is dependent on cell types and stretching force. Cyclic stretch $(0.3 \mathrm{~Hz}, 15 \%$ strain $)$ stimulates the expression of COL1A1 and CCN2/CTGF in human bladder smooth muscle cells [22]. In human chondrocytic HCS-2/8 cells, CTS $(0.16 \mathrm{~Hz}, 6 \%$ strain) increases CCN2/CTGF gene expression [23]. On the other hand, cyclic stretch $(0.16 \mathrm{~Hz}, 20 \%$ strain) decreases CCN2/CTGF expression in human skin fibroblasts [24]. In loading stress, the expressions of COL1A1, TGF- $\beta 1$, and CCN2/CTGF in rat soleus muscles are increased despite TGF- $\beta 1$ and CCN2/CTGF expressions in rat Achilles tendons are unaltered [25].

In the present study, we investigated the localization of CCN2/CTGF in human ACL tissues and evaluated its expression in response to mechanical stretch in human ACL-derived cells, in order to clarify the role of CCN2/CTGF in COL1A1 expression and the proliferation of ACL-derived cells.

\section{Materials and methods}

\subsection{Cells and cell culture}

Institutional Review Board approval was obtained for this study. Informed consents for this research were also obtained from patients. Human ACL fibroblasts were isolated from intact femur-ACL-tibia samples obtained at total knee arthroplasties in patients suffering from osteoarthritis $(n=6)$. Patients included five females and one male, with a mean age of 65 years (range, 62-72 years) at the time of surgery. Surrounding synovial tissues and attached bones were carefully removed from ACL samples. ACL-derived interface cells were isolated from 5-mm-segments of ACL-to-bone junctions (femoral and tibial interfaces) as described [15]. The remaining ligament was used as ACL-derived midsubstance cells. The ligament samples were minced, and incubated with collagenase (Sigma, 
St.Luis, MO) for $30 \mathrm{~min}$ at $37^{\circ} \mathrm{C}$ as described [26]. The mixtures were filtered through $70 \mu \mathrm{m}$ cell strainers (BD Biosciences, Bedford, MA). The flow through suspension was cultured in Dulbecco's Modified Eagle Medium (DMEM, Wako, Osaka, Japan) containing 10\% fetal bovine serum (HyClone, South Logan, UT) and 1\% penicillin/streptomycin (Sigma). Attached cells were incubated at $37^{\circ} \mathrm{C}$ in $5 \% \mathrm{CO}_{2}$ in a humidified atmosphere and were subcultured at a density of $2,500 \mathrm{cells} / \mathrm{cm}^{2}$ on uncoated polystyrene tissue culture dishes (BD Biosciences). The medium was changed every 3 days. Cells between passage 2 and 5 were used for experiments.

\subsection{RT-PCR and quantitative real-time PCR analysis}

Total RNA was isolated using ISOGEN reagent (Nippon Gene, Toyama, Japan). RNA samples obtained from tissue samples and cultured cells were reverse-transcribed with ReverTra Ace (Toyobo, Osaka, Japan). The cDNAs underwent PCR amplification in the presence of each set of specific primers using rTaq DNA polymerase (TaKaRa, Ohtsu, Japan). The following specific primer sets were used: 5'-CCACC CGAGT TACCA ATGAC-3' and 5'-GTGCA GCCAG AAAGC TCA-3' for

CCN2/CTGF; COL1A1 and glyceraldehyde-3-phosphate dehydrogenase (G3PDH) as described previously [27]. For all the RT-PCR fragments, the reactions were allowed to proceed for 30 cycles in an iCycler thermal cycler (Bio-Rad Laboratories, Hercules, CA). Quantitative real-time PCR analyses were performed by using LightCycler ST-300 instrument (Roche Diagnostics, Mannheim, Germany) and FastStart DNA Master SYBR Green I kit (Roche Diagnostics) according to the manufacturer's protocol. The cycle number crossing the signal threshold was selected in the linear part of the amplification curve. Amplification data of G3PDH were used for normalization.

\subsection{Cyclic tensile strain (CTS)}

Polydimethylsiloxane stretch chambers (STREX, Osaka, Japan) were coated with $50 \mu \mathrm{g} / \mathrm{ml}$ fibronectin (Chemicon, Temecula, CA). Midsubstance and interface cells were seeded onto stretch chambers, each having a culture surface of $2 \times 2 \mathrm{~cm}$, at the concentration of 50,000 cells/chamber. The cells were incubated on fibronectin-coated chambers for $24 \mathrm{~h}$ before stretching experiments. Uni-axial CTS (0.5 $\mathrm{Hz}, 10 \%$ strain) was applied using a STB-140 system (STREX) for 1, 2, and $4 \mathrm{~h}$ as described [15]. 
Non-stretched ACL cells cultured on stretch chambers were used as controls. RNAs and conditioned media were immediately collected after stretching experiments.

\subsection{Histology and immunohistochemistry}

To investigate the localization of CCN2/CTGF in human ACL, tissue samples obtained from surgical specimen under written informed consent were fixed with 4\% paraformaldehyde solutions for $24 \mathrm{~h}$ and were embedded in paraffin. Sections were cut into $5 \mu \mathrm{m}$-thickness and were stained with hematoxylin and eosin (HE) for standard light microscopy. Anti-CCN2/CTGF antibodies purified from rabbits by immunization with a synthetic peptide of CCN2/CTGF (RPCEA DLEEN IKKGK KCIRT) were used for immunohistochemical analyses as described $[5,28]$.

\subsection{Enzyme-linked immunosorbent assay (ELISA)}

The concentration of CCN2/CTGF in conditioned media was measured by using a sandwich ELISA system with two anti-human CCN2/CTGF monoclonal antibodies (MAb 8-64 and 8-86; mouse IgG1, Nichirei, Tokyo, Japan) as described previously [29]. In brief, samples were applied to ELISA strips coated with MAb 8-64 and incubated for 2 h. After 6 cycles of washing, horseradish peroxidase-conjugated MAb 8-86 was added, and the strips were incubated for $1 \mathrm{~h}$. The signals were developed by an enzymatic reaction with tetramethylbenzidine.

\subsection{CCN2/CTGF treatment and cell proliferation assay}

Cells were cultured on fibronectin-coated plates for $12 \mathrm{~h}$ in serum-free DMEM before the treatment with recombinant human CCN2/CTGF (BioVender, Candler, NC). CCN2/CTGF treatment (10 or 50 $\mathrm{ng} / \mathrm{ml}$ ) was performed for $4 \mathrm{~h}$ before the preparation of RNA samples. Cell proliferation assays were performed as described previously [30,31]. In brief, recombinant human CCN2/CTGF was added into serum-free DMEM at indicated concentrations. ACL cells $(5,000$ cells/well) were incubated for $48 \mathrm{~h}$ prior to addition of WST-1 (Roche Diagnostics) on 96-well plates. Optical density (OD) was measured by using a Model 550 microplate reader (Bio-Rad, Hercules, CA) at evaluation and control wavelengths of $450 \mathrm{~nm}$ and $630 \mathrm{~nm}$, respectively. Data obtained by subtracting 630-nm readings from 450-nm readings were used for evaluation. The mean value derived from four wells was computed and 
plotted.

\subsection{Statistical analysis}

All experiments were repeated at least three times and similar results were obtained. Data were expressed as mean values with standard deviations. Differences among groups were compared by using the Mann-Whitney $U$-test. Statistical significance was established at $p<0.05$.

\section{Results}

\subsection{Localization of CCN2/CTGF in human ACL}

We initially investigated the presence and distribution of $\mathrm{CCN} 2 / \mathrm{CTGF}$ in human ACL tissues. $\mathrm{CCN} 2 / \mathrm{CTGF}$ was observed in the ACL-to-bone interface, but was not in the midsubstance region by immunohistochemical analyses (Fig. 1C and D). Cells packaged in interface regions were stained by anti-CCN2/CTGF antibody (Fig. 1E and F), whereas CCN2/CTGF was not significantly detected in the ECM of tibial and femoral interface regions (Fig. 1E and F).

\subsection{Differences between interface and midsubstance cells in morphology and CCN2/CTGF production} Interface cells had different features from those of midsubstance cells. The morphology of interface cells in culture was typically characterized by a triangular shape (Fig. 2A). On the other hand, midsubstance cells had a fibroblastic morphology (Fig. 2A). Interestingly, the expression of CCN2/CTGF in respective cells showed different patterns from those in tissue samples, when they were brought into cell culture in vitro (Fig. 2B). Despite CCN2/CTGF was present in the tissues of interface region, RT-PCR analyses revealed that CCN2/CTGF expression was remarkably decreased in interface-derived cells, (Fig. 2B), which was efficiently recovered by CTS (Fig. 2C and D). CTS also increased CCN2/CTGF secretion from interface cells (Fig. 2F). On the other hand, CTS treatment did not increase the gene expression of CCN2/CTGF (Fig. 2C and D) and CCN2/CTGF secretion (Fig. 2F) in midsubstance cells. These results represent distinct biological characteristics of these 2 types of ACL-derived cells. Consistent with our previous findings [15], COL1A1 expression was enhanced by 
CTS treatment in both interface and midsubstance cells (Fig. 2C and E).

\subsection{Functional role of CCN2/CTGF in interface cells}

As more pronounced CTS-induced CCN2/CTGF expression was observed in interface cells, we further investigated the biological role of $\mathrm{CCN} 2 / \mathrm{CTGF}$ in ACL cells. Recombinant CCN2/CTGF treatment increased the expression of endogenous CCN2/CTGF gene only in interface cells (Fig. 3A and B). Surprisingly, COL1A1 expression was not influenced by CCN2/CTGF treatment alone in interface cells despite CCN2/CTGF stimulated COL1A1 expression in midsubstance cells (Fig. 3C). However, importantly, CCN2/CTGF significantly stimulated the proliferation of interface cells in a dose-dependent manner (Fig. 3D). These findings suggest that stretch-induced CCN2/CTGF might act as an activator for cellular proliferation, not for type I collagen production, in ACL-to-bone interface.

\section{Discussion}

CCN2/CTGF stimulates collagen gene expression in several types of cells such as chondrocytes, osteoblastic cells, periodontal ligament cells, and astrocytes [5-7,32,33]. Mechanical stretch also has a critical role in enhancing COL1A1 expression in ligament fibroblasts [15,21,34]. Yang et al. have described that cyclic stretch increases the expression of CCN2/CTGF and COL1A1 in bladder smooth muscle cells [22]. However, the relationship between CCN2/CTGF and stretch-induced COL1A1 expression remains unclear. We have previously reported that CTS induces higher increase of COL1A1 expression in human ACL interface cells, rather than in midsubstance cells [15]. In the present study, we demonstrated that CCN2/CTGF was localized in human ACL-to-bone interface (Fig. 1). CTS also promoted the expression of CCN2/CTGF in interface cells (Fig. 2). In addition, recombinant CCN2/CTGF stimulated endogenous CCN2/CTGF expression and cellular proliferation of interface cells, while COL1A1 expression was not affected by CCN2/CTGF treatment in the absence of mechanical stretch (Fig. 3). These findings suggest that stretch-mediated COL1A1 transactivation might not directly depend on stretch-induced CCN2/CTGF production in ACL 
interface cells. Considering the molecular property of CCN2/CTGF that promotes physiological process of tissue development and regeneration under multiple molecular interactions, CCN2/CTGF is expected to enhance the proliferation of interface cells in the absence of mechanical stretch, and type I collagen production of interface cells under stretched condition.

ACL-to-bone interface, characterized as fibrocartilaginous enthesis, shifts its characteristics from proliferative to hypertrophic zone of the growth plate during development $[12,19]$. Age-dependent changes in collagen deposition (from type II collagen to type X collagen) are observed at the interface [12]. Our previous study has indicated that cells in the interface zone of aged ACLs still express $\alpha 1$ (II) collagen (COL2A1) gene in three-dimensional tissue culture conditions (in vivo) [15]. In two-dimensional cell culture conditions (in vitro), non-stretched interface cells lose their potential to produce COL2A1 gene [15]. However, CTS induces COL2A1 re-expression in cultured interface cells [15]. The present study demonstrated that CTS treatment recovered the expression of CCN2/CTGF despite CCN2/CTGF expression was decreased in cultured interface cells (Fig. 2). In human chondrocytic HCS-2/8 cells, CCN2/CTGF expression is increased by CTS [23]; then CCN2/CTGF enhances the expression of the Col2a1 and aggrecan genes in rabbit growth plate and articular chondrocytes, as well as in HCS-2/8 cells [5,35]. These findings suggest that the fibrocartilaginous property of ACL-to-bone interface might be maintained by mechanical stretch-induced CCN2/CTGF.

Cellular responses to mechanical stress underlie many critical functions such as development, morphogenesis, hypertension, and wound healing [36]. In experimental studies, physiological force has been mimicked by various mechanical stimuli, including stretch, compression, shear stress, and bending torque [36]. Cell stretch would induce mechanical extension of cytoplasmic macromolecules, activation of ion channels, and phosphorylation of mechanotransducers [36-38]. However, cellular behavior is not uniform under comparable stretching condition in vitro. CCN2/CTGF expression is increased by cyclic stretch (no more than $15 \%$ strain) in bladder smooth muscle cells and chondrocytic cells $[22,23]$. On the other hand, $20 \%$ length of cellular stretch rather decreases CCN2/CTGF 
expression in skin fibroblasts [24]. In our experiments, the degree of stretching force (5-15\%) influenced the expression pattern of CCN2/CTGF and COL1A1 genes (Supplemental Fig.). The expression of CCN2/CTGF and COL1A1 was highly activated under $10 \%$ CTS condition in ACL interface cells (Supplemental Fig. and 2). Physiological stretching force might be important for inducing optimum production of CCN2/CTGF and ECM molecules to maintain ligament homeostasis. Cell morphology and actin cytoskeleton under various mechanical forces also regulate CCN2/CTGF expression [39]. In three-dimensional-cultured human lung fibroblasts, stretch-induced CCN2/CTGF expression is decreased by a specific inhibitor of RhoA/ROCK signaling pathway that modulates actin cytoskeleton [40]. Cyclic stretch-stimulated CCN2/CTGF promoter activity is suppressed by a $\underline{\text { RhoA/ROCK inhibitor in bovine bladder smooth muscle cells [41]. Mouse adipose-derived stromal }}$ cells produce higher levels of CCN2/CTGF and RhoA proteins in a low-density (spread) culture condition compared with a high-density-seeded condition [42]. On the other hand, CCN2/CTGF expression is not affected by a RhoA/ROCK inhibitor, but is down-regulated by a Rac1 inhibitor in mouse primary chondrocytes [43]. In our previous study, CTS activates integrin $\alpha \mathrm{V} \beta 3$-mediated stress fiber formation on fibronectin-coated chambers in human ACL fibroblasts [15]. From these findings, stretch-associated cytoskeletal tension, which is cooperatively modulated by several intracellular signaling pathways, may play an important role in CCN2/CTGF expression. Further studies will be required to clarify the relationships among stretch-activated signaling pathways, CCN2/CTGF expression, and ECM synthesis in ACL fibroblasts.

In conclusion, our results demonstrated that mechanical stretch stimulated the gene expression and synthesis of CCN2/CTGF in ACL-to-bone interface cells. Interface cell proliferation and COL1A1 expression in midsubstance cells were also enhanced by CCN2/CTGF treatments in the absence of CTS. These findings suggest that stretch-mediated CCN2/CTGF production might have a crucial role in regulating region-specific phenotypes of ACL-to-bone interface. 


\section{Acknowledgements}

We thank Ms. Motomi Hachioji, Dr. Aki Yoshida, and Dr. Eriko Aoyama for their kind cooperation.

We are also grateful to Dr. Nobuhiro Abe for providing tissue samples. This work was supported by Japan Society for the Promotion of Science (Nos. 19109008, 20791040, and 21592360), JSPS Fujita Memorial Fund for Medical Research, the Japanese Foundation for Research and Promotion of Endoscopy, Okayama Medical Foundation, Japan Orthopaedics and Traumatology Foundation (No. 225), and Child Health and Development from the Ministry of Health, Labor and Welfare.

\section{References}

1. B. Perbal, M. Takigawa (Eds.), CCN proteins: a new family of cell growth and differentiation regulators, Imperial College Press, London, 2005.

2. S. Ivkovic, B.S. Yoon, S.N. Popoff, et al., 2003. Connective tissue growth factor coordinates chondrogenesis and angiogenesis during skeletal development, Development 130 (2003) 2779-2791.

3. C.C. Chen, L.F. Lau, Functions and mechanisms of action of CCN matricellular proteins, Int. J. Biochem. Cell Biol. 41 (2009) 771-783.

4. T. Shimo, T. Nakanishi, T. Nishida, et al., Connective tissue growth factor induces the proliferation, migration, and tube formation of vascular endothelial cells in vitro, and angiogenesis in vivo, J. Biochem. 126 (1999) 137-145.

5. T. Nakanishi, T. Nishida, T. Shimo, et al., Effects of CTGF/Hcs24, a product of a hypertrophic chondrocyte-specific gene, on the proliferation and differentiation of chondrocytes in culture, Endocrinology 141 (2000) 264-273.

6. T. Nishida, T. Nakanishi, M. Asano, et al., Effects of CTGF/Hes24, a hypertrophic chondrocyte-specific gene product, on the proliferation and differentiation of osteoblastic cells in vitro, J. Cell. Physiol. 184 (2000) 197-206.

7. M. Asano, S. Kubota, T. Nakanishi, et al., Effect of connective tissue growth factor 
(CCN2/CTGF) on proliferation and differentiation of mouse periodontal ligament-derived cells, Cell Commun. Signal 3 (2000) 11.

8. T. Zantop, P.U. Brucker, A. Vidal, et al., Intraarticular rupture pattern of the ACL, Clin. Orthop. Relat. Res. 454 (2007) 48-53.

9. I.E. Wang, J. Shan, R. Choi, et al., Role of osteoblast-fibroblast interactions in the formation of the ligament-to-bone interface, J. Orthop. Res. 25 (2007) 1609-1620.

10. T. Furumatsu, M. Hachioji, K. Saiga, et al., Anterior cruciate ligament-derived cells have high chondrogenic potential, Biochem. Biophys. Res. Commun. 391 (2010) 1142-1147.

11. M. Benjamin, D. McGonagle, The anatomical basis for disease localisation in seronegative spondyloarthropathy at entheses and related sites, J. Anat. 199 (2001) 503-526.

12. I.E. Wang, S. Mitroo, F.H. Chen, et al., Age-dependent changes in matrix composition and organization at the ligament-to-bone insertion, J. Orthop. Res. 24 (2006) 1745-1755.

13. S. Thomopoulos, G.R. Williams, J.A. Gimbel, et al., Variation of biomechanical, structural, and compositional properties along the tendon to bone insertion site, J. Orthop. Res. 3 (2003) 413-419.

14. S. Thomopoulos, J.P. Marquez, B. Weinberger, et al., Collagen fiber orientation at the tendon to bone insertion and its influence on stress concentrations, J. Biomech. 39 (2006) 1842-1851.

15. T. Tetsunaga, T. Furumatsu, N. Abe, et al., Mechanical stretch stimulates integrin $\alpha \mathrm{V} \beta 3$-mediated collagen expression in human anterior cruciate ligament cells, J. Biomech. 42 (2009) 2097-2103.

16. D.R. Henshaw, E. Attia, M. Bhargava, J.A. Hannafin, Canine ACL fibroblast integrin expression and cell alignment in response to cyclic tensile strain in three-dimensional collagen gels, J. Orthop. Res. 24 (2006) 481-490.

17. V.B. Duthon, C. Barea, C. Abrassart, et al., Anatomy of the anterior cruciate ligament, Knee Surg. Sports Traumatol. Arthrosc. 14 (2006) 204-213.

18. A.J. Almarza, S.M. Augustine, S.L. Woo, Changes in gene expression of matrix constituents with 
respect to passage of ligament and tendon fibroblasts, Ann. Biomed. Eng. 36 (2008) 1927-1933.

19. K. Nawata, T. Minamizaki, Y. Yamashita, R. Teshima, Development of the attachment zones in the rat anterior cruciate ligament: changes in the distributions of proliferating cells and fibrillar collagens during postnatal growth, J. Orthop. Res. 20 (2002) 1339-1344.

20. D.L. Butler, Y. Guan, M.D. Kay, et al., Location-dependent variations in the material properties of the anterior cruciate ligament, J. Biomech. 25 (1992) 511-518.

21. S.G. Kim, T. Akaike, T. Sasagaw, et al., Gene expression of type I and type III collagen by mechanical stretch in anterior cruciate ligament cells, Cell Struct. Funct. 27 (2002) 139-144.

22. R. Yang, J. Amir, H. Liu, B. Chaqour, Mechanical strain activates a program of genes functionally involved in paracrine signaling of angiogenesis, Physiol. Genomics 36 (2008) 1-14.

23. T. Nishida, A. Maeda, S. Kubota, M. Takigawa, Role of mechanical-stress inducible protein Hcs24/CTGF/CCN2 in cartilage growth and regeneration: mechanical stress induces expression of Hcs24/CTGF/CCN2 in a human chondrocytic cell line HCS-2/8, rabbit costal chondrocytes and meniscus tissue cells, Biorheology 45 (2008) 289-299.

24. Y. Kanazawa, J. Nomura, S. Yoshimoto, et al., Cyclical cell stretching of skin-derived fibroblasts downregulates connective tissue growth factor (CTGF) production, Connect. Tissue Res. 50 (2009) 323-329.

25. K.M. Heinemeier, J.L. Olesen, F. Haddad, et al., Effect of unloading followed by reloading on expression of collagen and related growth factors in rat tendon and muscle, J. Appl. Physiol. 106 (2009) 178-186.

26. H. Date, T. Furumatsu, Y. Sakoma, et al., GDF-5/7 and bFGF activate integrin $\alpha 2$-mediated cellular migration in rabbit ligament fibroblasts, J. Orthop. Res. 28 (2010) 225-231.

27. T. Furumatsu, C. Shukunami, M. Amemiya-Kudo, et al., Scleraxis and E47 cooperatively regulate the Sox9-dependent transcription, Int. J. Biochem. Cell Biol. 42 (2010) 148-156.

28. S. Omoto, K. Nishida, Y. Yamaai, et al., Expression and localization of connective tissue growth factor (CTGF/Hcs24/CCN2) in osteoarthritic cartilage, Osteoarthritis Cartilage 12 (2004) 
$771-778$.

29. S. Kubota, K. Kawata, T. Yanagita, et al., Abundant retention and release of connective tissue growth factor (CTGF/CCN2) by platelets, J. Biochem. 136 (2004) 279-282.

30. T. Furumatsu, N. Yamaguchi, K. Nishida, et al., Endostain inhibits adhesion of endotherial cells to collagen I via $\alpha 2 \beta 1$ integrin, a possible cause of prevention of chondrosarcoma growth, $\mathrm{J}$. Biochem. 131 (2002) 619-626.

31. K. Saiga, T. Furumatsu, A. Yoshida, et al., Combined use of bFGF and GDF-5 enhances the healing of medial collateral ligament injury, Biochem. Biophys. Res. Commun. 402 (2010) 329-334.

32. S.J. Dangaria, Y. Ito, C. Walker, et al., Extracellular matrix-mediated differentiation of periodontal progenitor cells, Differentiation 78 (2009) 79-90.

33. R. Fuchshofer, M. Birke, U. Welge-Lussen, et al., Transforming growth factor- $\beta 2$ modulated extracellular matrix component expression in cultured human optic nerve head astrocytes, Invest. Ophthalmol. Vis. Sci. 46 (2005) 568-578.

34. D. Kaneko, Y. Sasazaki, T. Kikuchi, et al., Temporal effects of cyclic stretching on distribution and gene expression of integrin and cytoskeleton by ligament fibroblasts in vitro, Connect. Tissue Res. 50 (2009) 263-269.

35. T. Nishida, S. Kubota, T. Nakanishi, et al., CTGF/Hcs24, a hypertrophic chondrocyte-specific gene product, stimulates proliferation and differentiation, but not hypertrophy of cultured articular chondrocytes, J. Cell. Physiol. 192 (2002) 55-63.

36. A.W. Orr, B.P. Helmke, B.R. Blackman, M.A. Schwartz, Mechanisms of mechanotransduction, Dev. Cell 10 (2006) 11-20.

37. S.I. Sukharev, P. Blount, B. Martinac, et al., A large-conductance mechanosensitive channel in E. coli encoded by mscL alone, Nature 368 (1994) 265-268.

38. Y. Sawada, M. Tamada, B.J. Dubin-Thaler, et al., Force sensing by mechanical extension of the Src family kinase substrate p130Cas, Cell 127 (2006) 1015-1026. 
39. B. Chaqour, M. Goppelt-Struebe, Mechanical regulation of the Cyr61/CCN1 and CTGF/CCN2 proteins, FEBS J. 273 (2006) 3639-3649.

40. C. Schild, B. Trueb, Three members of the connective tissue growth factor family CCN are differentially regulated by mechanical stress, Biochim. Biophys. Acta 1691 (2004) 33-40.

41. B. Chaqour, R. Yang, Q. Sha, Mechanical stretch modulates the promoter activity of the profibrotic factor CCN2 through increased actin polymerization and NF- $\mathrm{BB}$ activation, J. Biol. Chem. 281 (2006) 20608-20622.

42. Y. Xu, D.R. Wagner, E. Bekerman, et al., Connective tissue growth factor in regulation of RhoA $\underline{\text { mediated cytoskeletal tension associated osteogenesis of mouse adipose-derived stromal cells, }}$ PLoS One 5 (2010) e11279.

43. A. Woods, D. Pala, L. Kennedy, et al., Rac1 signaling regulates CTGF/CCN2 gene expression via TGFbeta/Smad signaling in chondrocytes, Osteoarthritis Cartilage 17 (2009) 406-413. 


\section{Figure legends}

Fig. 1. CCN2/CTGF in human ACL. (A) Midsubstance region of ACL (HE). (B) ACL-to-bone interface (tibial insertion, HE). (C) CCN2/CTGF was not observed in midsubstance region by immunohistochemical analysis. (D) CCN2/CTGF was detected in the cells of ACL-to-bone interface (tibial insertion, brown). Insets in panels $\mathrm{C}$ and $\mathrm{D}$ show magnified images of the areas indicated by rectangles. (E and F) CCN2/CTGF was observed in/on the interface cells: E, tibial interface; F, femoral interface. Inset in panel $\mathrm{E}$ denotes a negative control in the absence of anti-CCN2/CTGF antibody. Arrowheads indicate the positive signals of CCN2/CTGF in femoral interface. Bars, 100 $\mu \mathrm{m}$.

Fig. 2. (A) Phase-contrast microscopic views of fibroblastic midsubstance cells and chondrocytic interface cells. Bars, $100 \mu \mathrm{m}$. (B) CCN2/CTGF expression in vivo and in vitro evaluated by RT-PCR analysis. Although CCN2/CTGF expression was detected in tissue samples of ACL interface, it was decreased in cultured interface cells. Effect of CTS on CCN2/CTGF and COL1A1 expression in ACL cells as evaluated by end-point RT-PCR (C) and by real-time PCR analyses (D). CTS $(0.5 \mathrm{~Hz}, 10 \%$ strain, $2 \mathrm{~h}$ ) enhanced CCN2/CTGF expression up to a 2.2-fold level of control only in interface cells (D, right panel), whereas COL1A1 expression was increased by CTS in both interface and midsubstance cells ( $\mathrm{C}$ and E). ELISA revealed that CTS treatment increased CCN2/CTGF secretion from interface cells (F). However, CTS did not increase CCN2/CTGF gene expression and CCN2/CTGF protein secretion in midsubstance cells (D and F). ${ }^{*} p<0.05$ compared with each 0-h-control.

Fig. 3. Effect of exogenous CCN2/CTGF on ACL cells. (A-C) Effect on CCN2/CTGF and COL1A1 expression evaluated by RT-PCR (A) and real-time PCR (B and C) analyses. Recombinant CCN2/CTGF enhanced the expression of endogenous CCN2/CTGF in interface cells (A and B). 
CCN2/CTGF treatment did not stimulate endogenous CCN2/CTGF expression in midsubstance cells (A and B). COL1A1 expression was not influenced by CCN2/CTGF treatment in interface cells despite CCN2/CTGF stimulated COL1A1 expression up to 2.4-fold level of control in midsubstance cells (C). (D) CCN2/CTGF stimulated the proliferation of interface cells in a dose-dependent manner. ${ }^{*} p<0.05$ compared with the index of $0 \mathrm{ng} / \mathrm{ml}$ control.

Supplemental Fig. Different patterns of gene expression in response to CTS. In midsubstance cells, COL1A1 expression was increased under 5 and $10 \%$ CTS conditions $(0.5 \mathrm{~Hz}, 2 \mathrm{~h})$. In interface cells, $10 \%$ CTS stimulated the expression of CCN2/CTGF and COL1A1 genes. On the other hand, higher stretching force ( $15 \%$ strain) decreased these gene expressions in both midsubstance and interface cells. 


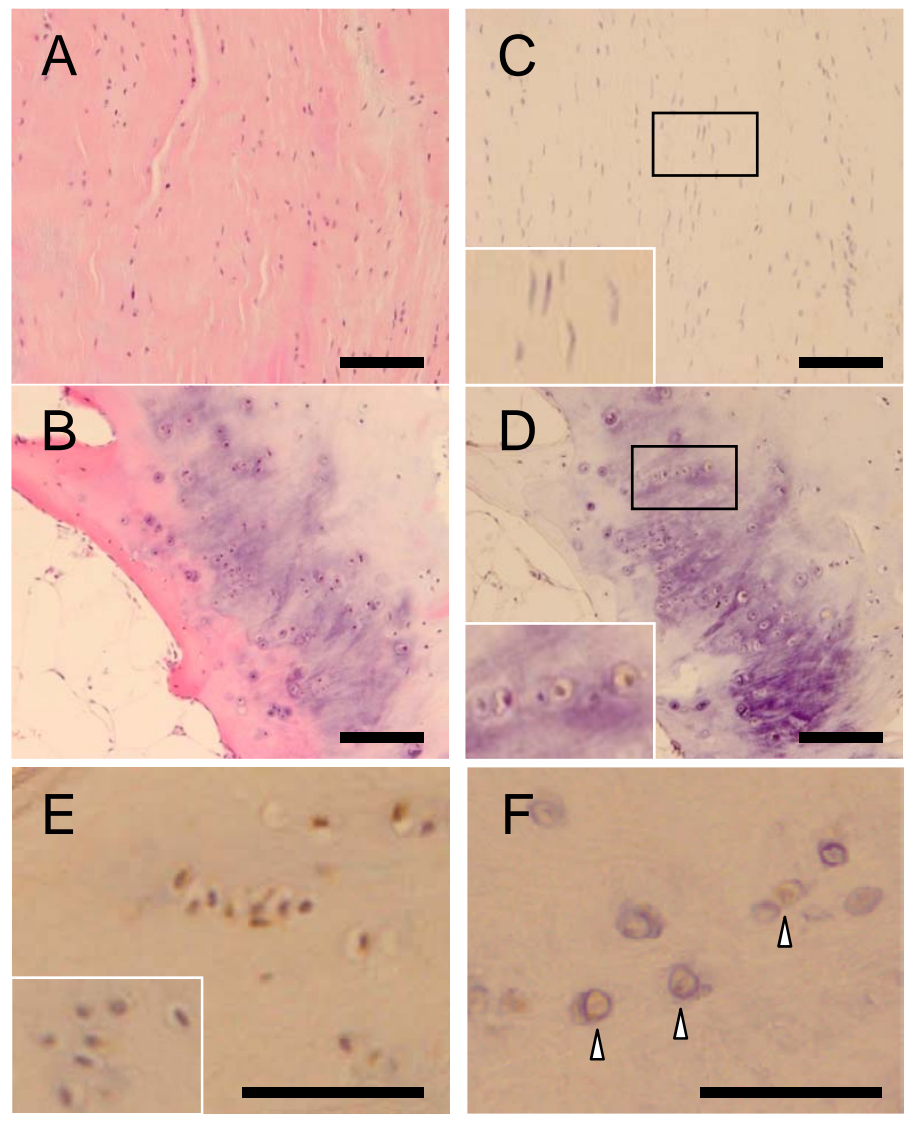

Figure 1.

Miyake et al. 


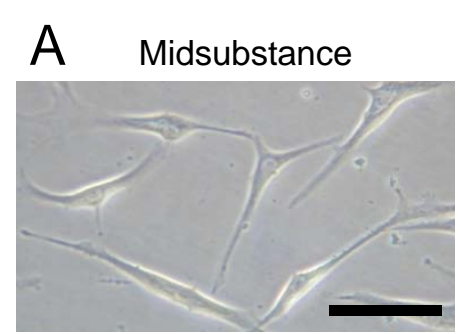

C
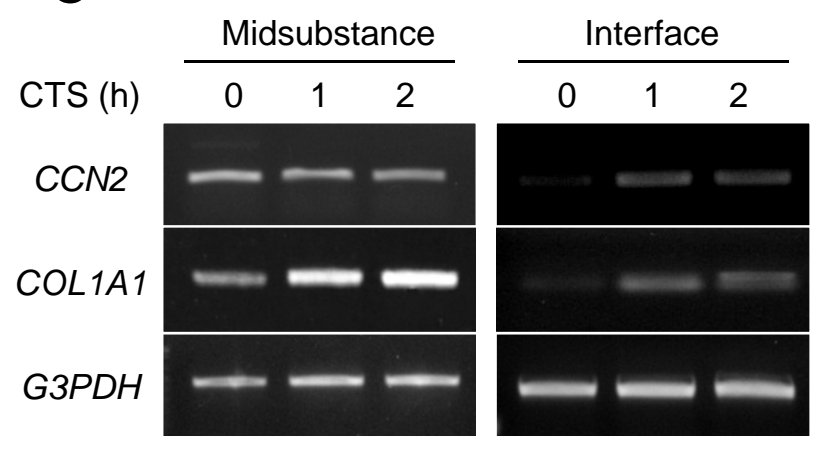

E Midsubstance

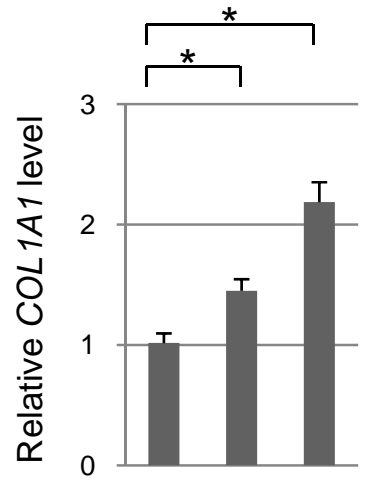

$\begin{array}{llll}\text { CTS (h) } & 0 & 1 & 2\end{array}$

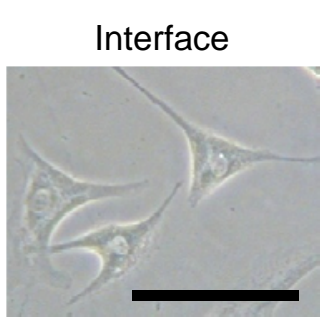

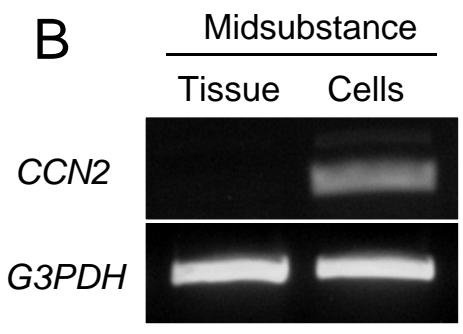

D Midsubstance

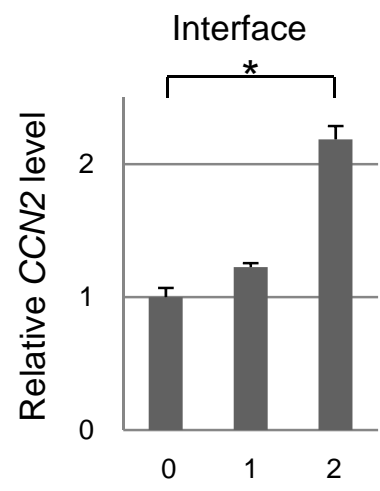

CTS (h)
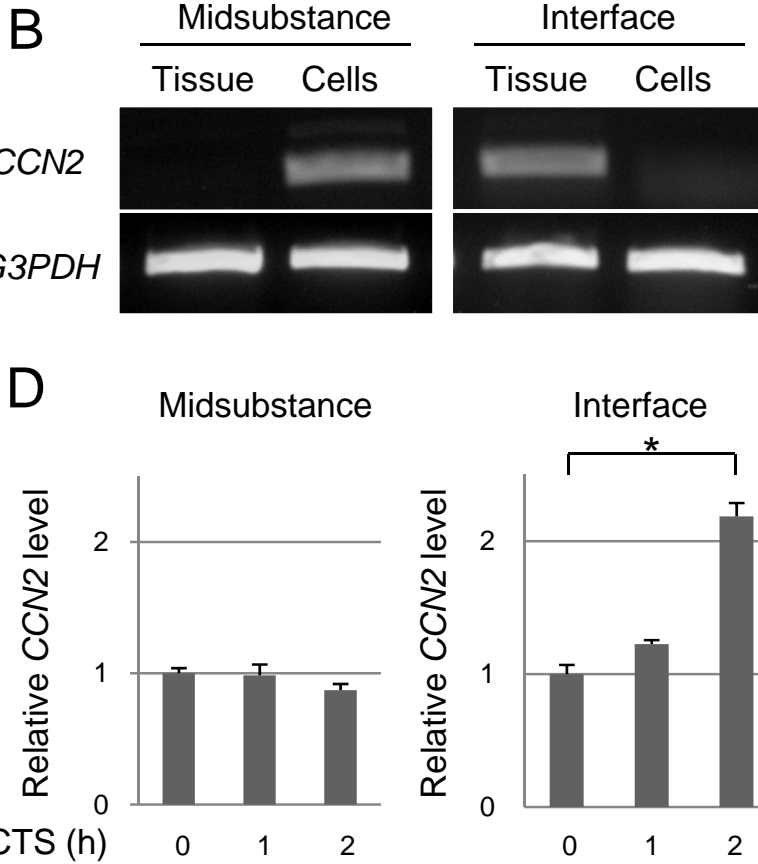

F Midsubstance

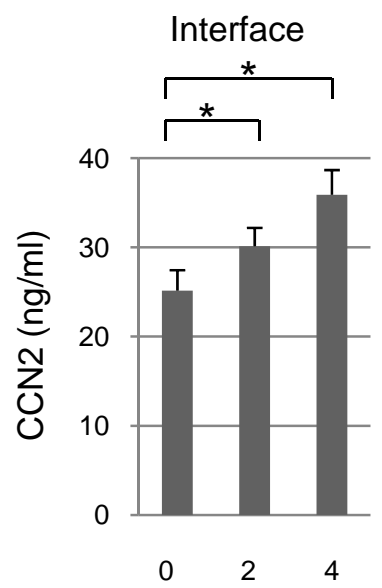

Figure 2.

Miyake et al. 

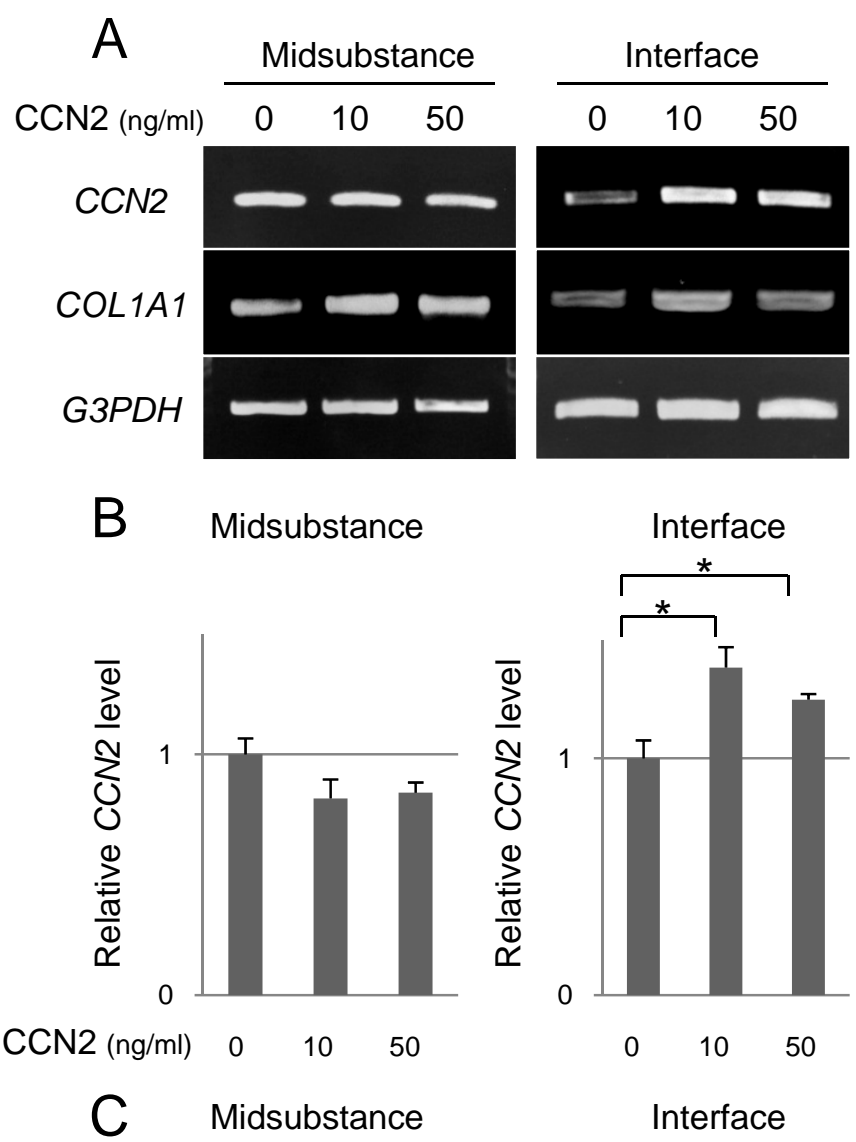

C Midsubstance
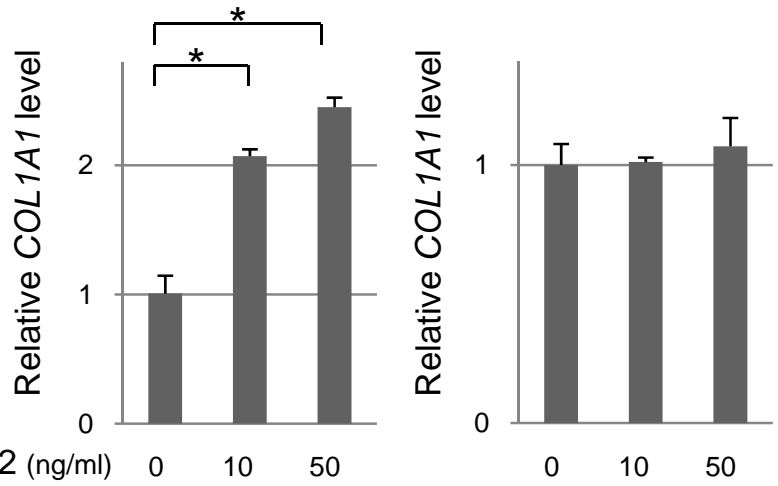

D Midsubstance

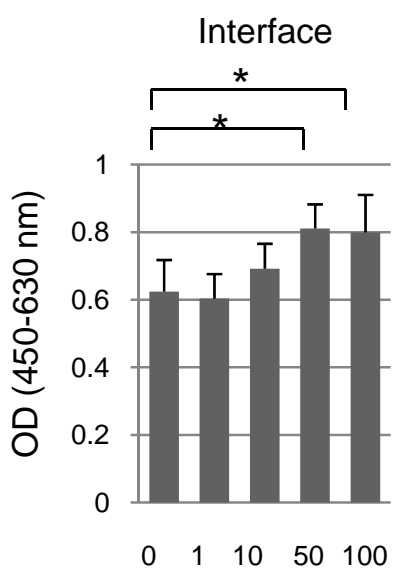

CCN2 (ng/ml) $0 \quad 1 \quad 10 \quad 50100$

Figure 3.

Miyake et al. 


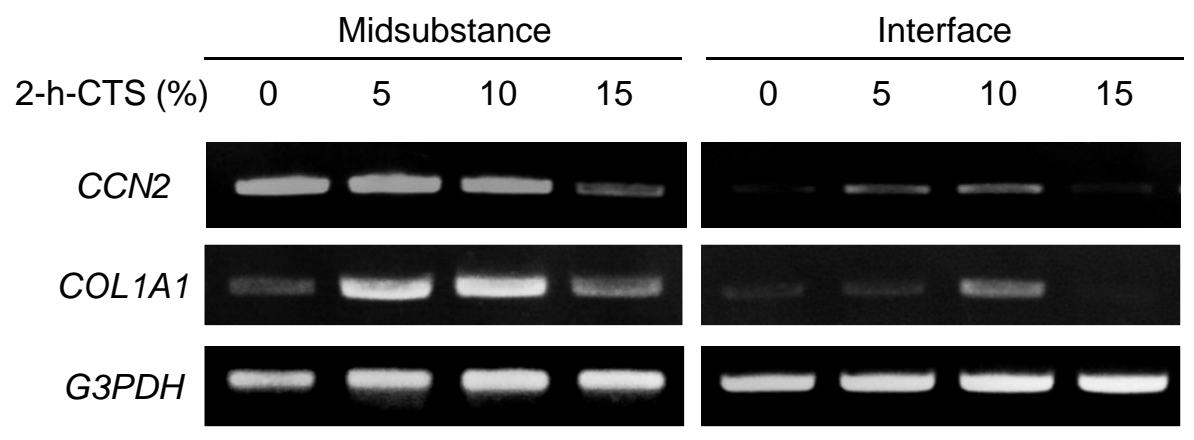

\section{Supplemental Figure Miyake et al.}

